\title{
A rare variation of the incomplete coeliac trunk
}

\author{
J.K. Lee' ${ }^{1}$, S.R. Kang ${ }^{1}$, J. Kim², S.P. Yoon², 3 \\ ${ }^{1}$ Medical Course, Medical School, Jeju National University, Jeju-Do, Republic of Korea \\ 2Department of Anatomy, Medical School, Jeju National University, Jeju-Do, Republic of Korea \\ 3 Institute of Medical Science, Jeju National University, Jeju-Do, Republic of Korea
}

[Received: 2 April 2015; Accepted: 10 May 2015]

During the routine dissection course, we found a rare variation of the incomplete coeliac trunk in an 87-year-old Korean male cadaver. The left gastric artery, the splenic artery and the hepatomesenteric trunk arose independently from the abdominal aorta. The detailed branching pattern of this case could not be classified by classic classification systems, but belongs to type III' of Morita's classification. The accurate embryological knowledge on the arterial variations is important both to anatomists and to clinicians. (Folia Morphol 2016; 75, 1: 122-124)

Key words: arterial variations, coeliac trunk, primitive splanchnic branches

\section{INTRODUCTION}

The foetal vascular system consists of a pair of dorsal aortic anlage. Each anlage gives off ventral segmental branches supplying the gastrointestinal tract. The 2 dorsal aortas fuse together to form a single dorsal aorta and the pairs of ventral segmental arteries fuse in the midline by the middle of $5^{\text {th }}$ week [13]. Four primitive splanchnic branches arise from the dorsal aorta in the middle region of the embryo and form the coeliac trunk and the superior mesenteric artery (SMA). These branches are connected to the ventral longitudinal anastomotic channels in early human embryos. Retention or disappearance of parts of this primitive arterial plexus could give rise to anomalous variations of the coeliac trunk and the SMA $[10,13,17]$.

Numerous reports on vascular distributional patterns including their origins, numbers, and even routes have been published and classified [1-3, 7-9], since understanding the distribution of the coeliac trunk and the SMA is essential for medical practice. The existence of a hepatomesenteric trunk has been reported with the highest incidence of 3.5\% [2], which is also true in Koreans as appeared in $2.96 \%$ [15]. On the other hand, the other association with a par- ticular pattern of the coeliac trunk has been occasionally reported on the detailed branching patterns in this region. Herein, we report a rare arterial variation of the left gastric artery, the splenic artery and the hepatomesenteric trunk independently arisen from the abdominal aorta.

\section{CASE REPORT}

During a routine dissection at Jeju National University Medical School in 2014, we found a rare arterial variation of the incomplete coeliac trunk in an 87-year-old Korean male cadaver, whose cause of death was 'cholangiocarcinoma'.

The classic coeliac trunk was not identified, but the left gastric artery, the splenic artery and the hepatomesenteric trunk independently arose from the abdominal aorta (Fig. 1). At the level of the 12th thoracic vertebral body, the left gastric artery arose from the abdominal aorta just on the left side from the median line of its anterior wall. The external diameter of this artery at its origin was $2.0 \mathrm{~mm}$, and it ran laterally to reach the lesser curvature of the stomach.

At the same level just below the origin of the left gastric artery, the splenic artery arose from the ab- 


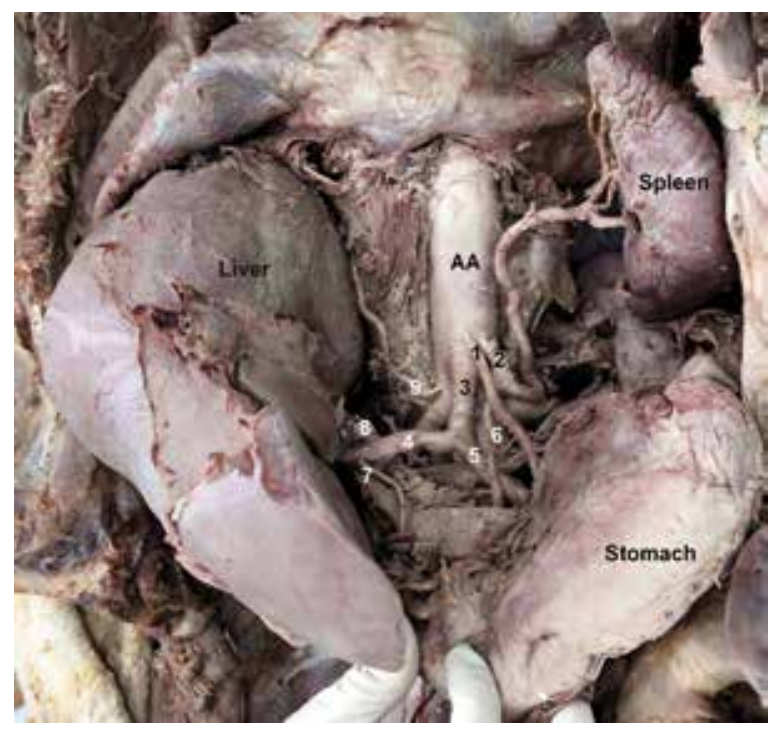

Figure 1. Photograph of this case. The left gastric artery (1), the splenic artery (2) and the hepatomesenteric trunk (3) arose from the abdominal aorta (AA) independently. And then the hepatomesentric trunk was divided into the common hepatic artery (4) and the superior mesenteric artery (5); 6 - the dorsal pancreatic artery; 7 - the right gastric artery; 8 - the gastroduodenal artery; 9 - the right inferior phrenic artery.

dominal aorta on the median line of its anterior wall just on the right side of the origin of the left gastric artery. The external diameter of this artery at its origin was $5.0 \mathrm{~mm}$, and it ran laterally to reach the spleen. In the course of this artery, it had a main pancreatic branch (dorsal pancreatic artery) which ran towards the posteroinferior region of the pancreas.

The hepatomesenteric trunk arose from the anterior wall of the abdominal aorta at the inferior part of the first lumbar vertebral body, which was $11.5 \mathrm{~mm}$ distal from the origin of the left gastric artery. The external diameter of this trunk at its origin was $6.5 \mathrm{~mm}$, and it was divided into the common hepatic artery (the external diameter at its origin, $5.5 \mathrm{~mm}$ ) and the SMA (the external diameter at its origin, $6.0 \mathrm{~mm}$ ). The common hepatic artery ran upward and laterally, and gave off the right gastric artery (the external diameter at its origin, $2.0 \mathrm{~mm}$ ) and the gastroduodenal artery (the external diameter at its origin, $4.8 \mathrm{~mm}$ ) respectively.

\section{DISCUSSION}

Numerous resembled anatomic variations in this region were classified by previous classical studies $[1,8,9]$, and clinically modified $[2,3,7,15,18]$. The present case can be considered to be quite similar to the previous classification, which consists of the hepatomesenteric trunk and the gastrosplenic trunk. However, this case is not suitable for detailed classification and is necessary to study deep into a mechanism of embryological arterial development although this case could be included in a certain classification [18] which has flexible subcategories - no coeliac trunk.

From embryological point of view, Morita [10] classified a variety of arrangements of origin of the coeliac trunk and the SMA as 5 types and 15 forms. The Morita's classification for the present case is type III': the left gastric artery, the splenic artery and the hepatomesenteric trunk (Fig. 2), which has been previously reported only 1 case [14]. Similar variations with a special reference to the hepatomesenteric trunk were reported as Morita's type II' - the hepatosplenomesenteric trunk and the left gastric artery [16]

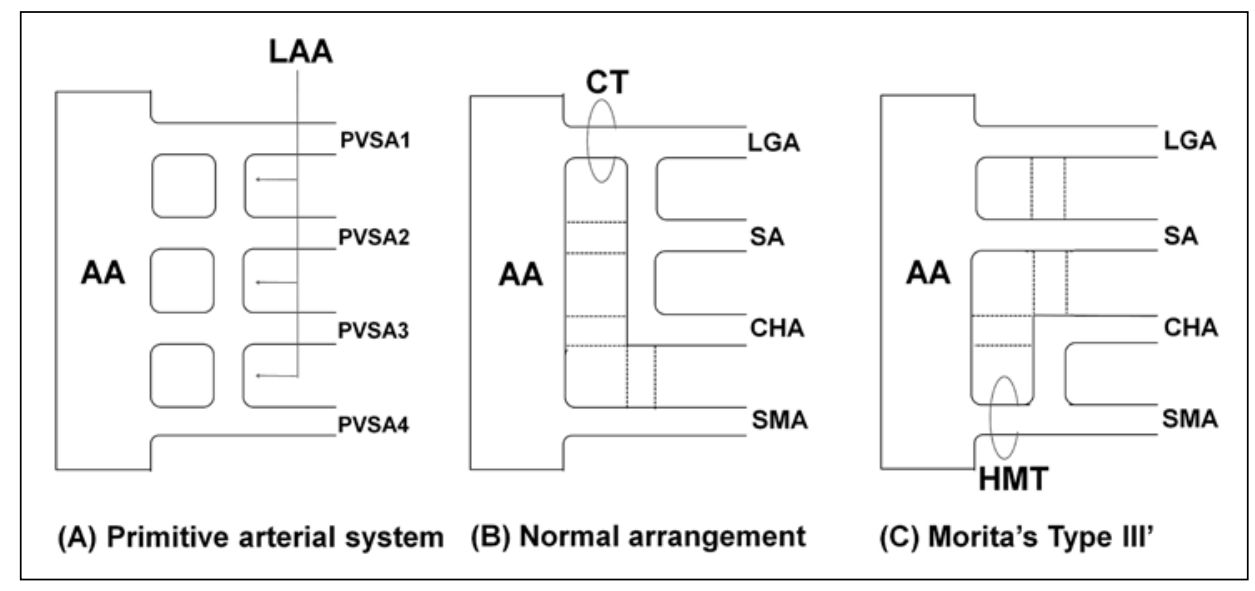

Figure 2. Schematic diagram of developmental pattern based on Tandler's embryogenic hypothesis (A, B) and type III' of Morita's classification (C); AA — abdominal aorta; CHA — the common hepatic artery; CT — the coeliac trunk; HMT — the hepatomesenteric trunk; LAA — the longitudinal anastomotic arteries; LGA — the left gastric artery; PVSA — the primitive ventral splanchnic arteries; SA — the splenic artery; SMA — the superior mesenteric artery. 
and type IV' - the gastrosplenic trunk and the hepatomesenteric trunk [4-6, 12]. Furthermore, an unclassified case of the common hepatic artery and inferior mesenteric artery arising from the SMA was also reported [11].

The limitation of this case is the portal area, which was unclear due to the previous operation for cholangiocarcinoma. The variant common hepatic artery arising from the SMA is more important to clinicians than the lienosplenic trunk or no trunk, although the course of the common hepatic artery as pre-/ /retro-portal vein has been suggested an important consideration for clinicians [15]. In this context, Morita's type III' might have been missed and taken as a variant of the gastrosplenic trunk. In addition, this rarity on Morita's type III' might also be caused by clinging to an old but distinct literary of Adachi [1], in which Morita's type III' could be regarded as a variant of type $\mathrm{V}$ (form 23) or type VI (form 24) in Adachi's classification.

\section{CONCLUSIONS}

Taken together, we found a rare arterial anomaly of the left gastric artery, the splenic artery and the hepatomesenteric trunk independently arising from the abdominal aorta. The knowledge of these arterial variations throughout the embryological base is essential for the clinicians as well as anatomists.

\section{REFERENCES}

1. Adachi B (1928) Das Arteriensystem der Japaner. Bd 1. Vol. 2. Die Kaiserlich Japanische Universität zu Kyoto, Kyoto, pp. 11-74.

2. Chen H, Yano R, Emura S, Shoumura S (2009) Anatomic variation of the celiac trunk with special reference to hepatic artery patterns. Ann Anat, 191: 399-407.

3. Hiatt JR, Gabbay J, Busuttil RW (1994) Surgical anatomy of the hepatic arteries in 1000 cases. Ann Surg, 220: 50-55.

4. limura A, Oguchi T, Shibata M, Takahashi T (2007) An anomalous case of the hepatic artery arising from the superior mesenteric artery. Okajimas Folia Anat Jpn, 84: 61-65.
5. Kahraman G, Marur T, Tanyeli E, Yildirim M (2001) Hepatomesenteric trunk. Surg Radiol Anat, 23: 433-435.

6. Kardile PB, Ughade JM, Ughade MN, Dhende A, Ali SS (2013) Anomalous origin of the hepatic artery from the hepatomesenteric trunk. J Clin Diagn Res, 7: 386-388.

7. Koops A, Wojciechowski B, Broering DC, Adam G, Krupski-Berdien G (2004) Anatomic variations of the hepatic arteries in 604 selective celiac and superior mesenteric angiographies. Surg Radiol Anat, 26: 239-244.

8. Lippert H, Pabst R (1985) Arterial variations in man. Classification and frequency. JF Bergmann Verlag, Munchen, pp. 30-41.

9. Michels NA (1956) Blood supply and anatomy of the upper abdominal organs. JB Lippincott Co., Philadelphia and Montreal, pp. 139-141.

10. Morita M (1935) Reports and conception of three anomalous cases in the area of the celiac and the superior mesenteric arteries. Igaku Kenkyu, 9: 1993-2006.

11. Osawa T, Feng XY, Sasaki N, Nagato $S$, Matsumoto $Y$, Onodera M, Nara E, Fujimura A, Nozaka Y (2004) Rare case of the inferior mesenteric artery and the common hepatic artery arising from the superior mesenteric artery. Clin Anat, 17: 518-521.

12. Padin JM, Ramisch DA, Maraschio M, Samame J, Farinelli PA, Pfaffen G, Barros Schelotto P, Gondolesi GE (2013) Intrapancreatic common hepatic artery arising from the superior mesenteric artery, a challenging anatomic variation in a multiorgan harvesting. Transplant Proc, 45: 820-823.

13. Pansky B (2015) Development of the arterial system. Review of medical embroyology. Available from: http://discovery.lifemapsc.com/library/review-of-medical-embryology/ chapter-123-development-of-the-arterial-system.

14. Saga T, Hirao T, Kitashima S, Watanabe K, Nohno M, Araki Y, Kobayashi S, Yamaki K (2005) An anomalous case of the left gastric artery, the splenic artery and hepatomesenteric trunk independently arising from the abdominal aorta. Kurume Med J, 52: 49-52.

15. Song SY, Chung JW, Yin YH, Jae HJ, Kim HC, Jeon UB, Cho BH, So YH, Park JH (2010) Celiac axis and common hepatic artery variations in 5002 patients: systematic analysis with spiral CT and DSA. Radiology, 255: 278-288.

16. Sridhar Varma K, Pamidi N, Vollala VR, Bolla SR (2010) Hepato-spleno-mesenteric trunk: a case report. Rom J Morphol Embryol, 51: 401-402.

17. Tandler J (1904) Über die Varietäten der Arteria coeliaca und deren Entwicklung. Anat Hefte, 25: 473-500.

18. Vandamme JP, Bonte J (1985) The branches of the celiac trunk. Acta Anat (Basel) 122: 110-114. 